Théologiques

Théologiques

\title{
Frère François et ses frères, les animaux Évocations
}

\section{Richard Bergeron}

Volume 10, numéro 1, printemps 2002

Les animaux dans la conscience humaine

URI : https://id.erudit.org/iderudit/008158ar

DOI : https://doi.org/10.7202/008158ar

Aller au sommaire du numéro

\section{Éditeur(s)}

Faculté de théologie de l'Université de Montréal

ISSN

1188-7109 (imprimé)

1492-1413 (numérique)

Découvrir la revue

Citer cet article

Bergeron, R. (2002). Frère François et ses frères, les animaux : évocations. Théologiques, 10(1), 109-129. https://doi.org/10.7202/008158ar
Résumé de l'article

Cet article propose une série d'évocations sur le rapport de François d'Assise avec les animaux, rapport qui est abordé ici à partir du récit du Sermon aux oiseaux qui s'impose par son ancienneté, son autorité et sa popularité. Le récit contient les principaux thèmes évoqués dans la cinquantaine d'histoires d'animaux rencontrés dans leLégende franciscaine : innocence originelle, harmonie, soumission des animaux, maîtrise des bêtes en soi-même, valeurs symboliques. Le rapport de François aux animaux n'est ni romantique, ni écologique mais mystique. Ce n'est qu'après avoir passé à travers les exigences d'une rude ascèse et d'une dépossession radicale qu'il redécouvre la portée épiphanique de la nature et le lien fraternel qui l'unit aux choses et aux bêtes. L'expérience érémitique a été le premier déclenchement d'un tel sentiment de fraternité avec les bêtes qui a trouvé dans le vita apostolica un second lien d'inscription. Le rapport de François aux animaux doit être compris sur l'horizon de son fameux Cantique des créatures qui est l'expression la plus puissante de sa vision mystique de l'interconnexion de toutes réalités créées. 


\title{
Frère François
}

\section{et ses frères, les animaux Évocations}

\author{
Richard BERgERON \\ Professeur émérite \\ Faculté de théologie \\ Université de Montréal
}

Qui veut parler sérieusement de François d'Assise se sent immédiatement piégé par l'épineuse question des sources qui n'est toujours pas résolue; ce qui n'entraîne pas pour autant l'impossibilité de tracer un portrait véridique du genre d'homme qu'il a été, du genre de discours qu'il a tenu et du genre d'actions qu'il a posées. Jusqu'à tout récemment, les nombreuses images, complémentaires sinon contradictoires, qui circulent sur le Poverello, sont généralement tirées des hagiographies ou légendes franciscaines plutôt que de ses propres écrits qui ont hélas! été longtemps négligés ${ }^{1}$. Sans nier la validité du recours aux biographies primitives comme intermédiaires utiles pour prendre contact avec l'esprit de l'homme d'Assise, force nous est de reconnaître que la figure qui émerge des écrits de François offre une configuration bien différente de celle qui est traditionnellement dessinée à partir des légendes. Cela est vrai, entre autres, du rapport de François avec les animaux.

Les hagiographies primitives, qui abondent en histoires d'animaux, présentent un François amant de la nature qui parle aux bêtes

1. François D'Assise, Écrits / texte latin de l'édition K. Esser; introduction, traduction, notes et index par T. Desbonnets et al. (Sources chrétiennes 285), Paris, Éditions du Cerf, 1981. On y retrouve, entre autres: La Première Règle, Le Cantique des créatures, les deux Lettres aux fidèles, la Salutation des vertus et l'Exhortation à la louange de Dieu - textes dont il sera question dans cet article. 
et les humanise. Sous l'influence de ces récits, on a fait de François le patron des scouts et des écologistes, un rêveur romantique, un mystique panthéiste. Tendance à le conformer à ses attentes personnelles et aux préconceptions modernes pour en faire un "freak» médiéval qui n'a pas poussé jusqu'au bout son amour de toute vie en devenant végétarien. Au contraire des légendes, les écrits de François observent un surprenant silence sur ce point. Il n'y est jamais question d'animaux, sauf pour dire que les frères doivent leur être soumis, qu'il leur interdit explicitement d'aller à cheval et de ne posséder "aucune bête en aucune manière, ni chez eux, ni chez les autres, ni de toute manière» (Première Règle 15,1). Même le Cantique des créatures, oh surprise!, ignore les animaux. Et pourtant... existait-il un lieu plus propice à leur évocation?

Les premiers biographes ont-ils voulu délibérément transformer sinon déformer la figure de François selon leurs tendances spirituelles, ou tout simplement mettre en relief un aspect réel de sa vie et de sa personnalité ? Je n'entends pas ici répondre à cette question. M'est avis cependant qu'il leur était légitime, pour peindre le portrait du saint, d'utiliser le genre littéraire "histoires d'animaux» dont la legenda sanctorum regorgeait depuis les Pères du désert et qui était toujours populaire au Moyen-Âge, surtout chez les moines irlandais. Le thème des animaux a toujours offert un jeu complexe d'images puissantes servant à évoquer aussi bien la lutte spirituelle et le retour à l'innocence que l'harmonie cosmique et l'envol mystique. Aussi toute religion a-t-elle développé son propre bestiaire selon le spécifique de la spiritualité qu'elle propose.

La question demeure vive: François a-t-il été l'ami des animaux que présentent les premiers biographes? Quel a été son rapport à la nature et aux bêtes? Quel sens a-t-il donné à ce rapport et quelle place occupe-t-il dans sa spiritualité ? Des études savantes ont été menées sur les sources par nombre de spécialistes de la question franciscaine. À côté des Armstrong, Leclerc, Monti, Marini, Sorrell²,

2. E.A. Armstrong, Saint-Francis: Nature Mystic, Berkeley, University of California Press, 1973; É. Leclerc, Le Cantique des créatures, Paris, Desclée 
je ne puis prétendre à quelque contribution scientifique digne d'intérêt. Je me contenterai d'une évocation tirée à la fois de mon imagination, de ma longue fréquentation des écrits franciscains, de mon amour pour l'homme d'Assise et de mon attachement à sa spiritualité. Cette évocation est construite autour du récit du Sermon aux oiseaux, tel qu'il se présente en Celano, récit qui possède une autorité unique à cause de la date de sa composition et de la référence au témoignage explicite de François lui-même et de ses compagnons.

\section{1}

De toutes les histoires d'animaux qui ponctuent la légende franciscaine, la prédication aux oiseaux est sûrement celle qui a frappé avec le plus de vivacité l'imagination populaire. La scène a été représentée par de nombreux artistes qui ont épuisé leur imagination à dessiner des oiseaux de rêve dans un décor idyllique. Ils dépeignent François tantôt penché vers les oiseaux regroupés sur le sol, tantôt la tête levée vers les branches d'un arbre. C'est le seul événement représentant François avec les animaux qui soit peint par Giotto dans la Basilique majeure à Assise. La légende remonte aux origines du mouvement franciscain; elle faisait probablement partie des histoires merveilleuses qui circulaient déjà sur le Poverello de son vivant. La tradition orale a été consignée dans six versions écrites, dont la plus primitive est la Vita prima de Celano ${ }^{3}$, en 1228, reprise par Bonaventure dans sa Legenda Major ${ }^{4}$, en 1263, et la plus tardive les

de Brower, 1970; V. Monti, François d'Assise et les animaux, d'après ses premières biographies. Mémoire de Maîtrise, Université de Montréal, 1988; A. Marini, Sorores alaudae, Francesco d'Assisi, il creato, gli animali, Assise, Ed. Porziuncula, 1989; R.D. Sorrell, St. Francis of Assisi and Nature, Oxford, Oxford University Press, 1988.

3. Thomas de Celano, Vie de saint François d'Assise / traduction française, introduction et notes de D. Vorreux, Paris, Éditions franciscaines, 1952. Sera cité sous son titre latin, Vita prima.

4. Bonaventure, Vie de saint François d'Assise / traduite du latin avec une introduction par M.J. Fagot, Paris, Art catholique, 1925. 
Fioretti $^{5}$ (vers 1380), qui reprennent pour l'essentiel le récit des Actus beati Francisci ${ }^{6}$ (vers 1328). Le texte de Celano, plus bref, représente la version officielle commandée et approuvée par le pape Grégoire IX deux ans après la mort de François. Celle des Fioretti, non officielle, représente une branche de la tradition qui dériverait du Frère Masséo, compagnon de la première heure, mort en 1280. Cette tradition serait demeurée longtemps sous la forme orale. La version des Fioretti, plus longue, ne manque pas d'intérêt historique malgré son caractère tardif.

Voici la scène, telle que décrite par Celano (Vita prima, 58).

Après l'arrivée de nouveaux frères, le bienheureux François prit la route et suivit la vallée de Spolète. Comme il approchait de Bevagna, il rencontra, rassemblés par bandes entières, des oiseaux de tous genres: ramiers, corneilles et freux. Sitôt qu'il les vit, il planta là ses compagnons et courut vers les oiseaux.

Son amour était si débordant qu'il témoignait même aux créatures inférieures et privées de raison une grande affection et une grande douceur. Arrivé tout près d'eux, il constata que les oiseaux l'attendaient; il leur adressa le salut habituel, s'émerveilla de ce qu'ils ne se fussent pas envolés comme ils font d'habitude, leur dit qu'ils devaient écouter la parole de Dieu et les pria humblement d'être attentifs.

Il leur dit, entre autres choses:

"Mes frères les oiseaux, vous avez bien sujet de louer votre créateur et de l'aimer toujours; Il vous a donné des plumes pour vous vêtir, des ailes pour voler et tout ce dont vous avez besoin pour vivre.

De toutes les créatures de Dieu, c'est vous qui avez meilleure grâce; il vous a dévolu pour champ l'espace et sa simplicité;

Vous n'avez ni à semer, ni à moissonner; il vous donne le vivre et le couvert sans que vous ayez à vous en inquiéter.»

À ces mots, rapportent le saint lui-même et ses compagnons, les oiseaux exprimèrent à leur façon une admirable joie; ils allongeaient le

5. I Fioretti, les petites fleurs de la vie du petit pauvre de Jésus-Christ saint François d'Assise / traduction, introduction et notes par A. Goffin (Science et religion 516-517), Paris, Bloud, [s.d.].

6. Actus beati Francisci et sociorum eius / édité par M. Bigaroni e G. Boccali, Assisi, Edizioni Porziuncola, 1988. 
cou, déployaient les ailes, ouvraient le bec et regardaient attentivement. Lui allait et venait parmi eux, frôlant de sa tunique et leurs têtes et leurs corps. Finalement, il les bénit, traça sur eux le signe de la croix et leur permit de s'envoler. Il reprit la route avec ses compagnons et, délirant de joie, rendit grâce à Dieu qui est ainsi reconnu et vénéré de toutes ses créatures.

Il n'était pas simple d'esprit, mais il avait la grâce de la simplicité. Aussi s'accusa-t-il de négligence pour n'avoir pas encore prêché aux oiseaux puisque ces animaux écoutaient avec tant de respect la parole de Dieu. Et à partir de ce jour, il ne manquait pas d'exhorter tous les oiseaux, tous les animaux, les reptiles et même les créatures insensibles, à louer et aimer le Créateur, car à l'invocation du nom du Sauveur, il faisait tous les jours l'expérience de leur docilité.

Ce récit reprend les principaux thèmes que les biographies primitives développent dans leurs histoires d'animaux. François s'adresse aux oiseaux. Nous savons que, sur la cinquantaine d'histoires d'animaux que racontent les hagiographes, une vingtaine mettent des oiseaux en scène: faucons, hirondelles, tourterelles, rossignols, poules d'eau, alouettes, corneilles. Les récits racontent cette merveilleuse connivence de François avec les oiseaux. À l'Alverne, le saint apprivoise le faucon qui lui sert de réveille-matin. Il libère les tourterelles qu'on amène au marché, il invite la poule d'eau à reprendre sa liberté, il domestique des tourterelles, il convertit le renard qui dévore les poules d'un pauvre fermier, il remet à l'eau des poissons capturés et leur commande de ne plus se laisser prendre; il chante des cantiques spirituels en alternance avec le rossignol. À plusieurs occasions, il fait taire hirondelles et autres volatiles qui empêchent ses auditeurs d'entendre sa prédication, ou qui rendent impossible la récitation des psaumes ou encore qui le dérangent dans sa méditation ou dans la dictée de sa Règle à frère Léon. Il se lie d'amitié avec un faisan qui ne veut plus le quitter; il affectionne particulièrement l'humble alouette à capuchon qui vint à sa mort chanter "une plainte joyeuse » et un "alléluia triste». Que dire de tous ces oiseaux qui, au cours des visites de François à l'Alverne, viennent survoler son 
refuge et chanter tour à tour leurs mélodies annonciatrices des stigmates! Que dire encore de son fol souhait que l'on décrète une loi spéciale obligeant maires de villages et seigneurs de châteaux à répandre le jour de Noël des graines le long des routes pour que les oiseaux aient de la nourriture en abondance en ce jour béni entre tous! Et l'histoire du canard qui attaque le loup pour lui faire lâcher le bébé qu'il tenait dans sa gueule! Et cette famille de rouges-gorges qui vivent avec les frères dans le couvent «non comme des invités, mais comme les membres de la famille». Et ces hirondelles qui déposent une goutte d'eau fraîche sur les lèvres desséchées de François! Y a-t-il tableau plus idyllique que cette multitude d'oiseaux qui viennent l'accueillir lors de sa première montée à l'Alverne? Chantant, battant des ailes, ils se posent sur la tête du saint et sur ses épaules, ou encore sur ses genoux, ses bras et sa poitrine.

Dans toutes ces histoires pour enfants et adultes, François vit en parfaite harmonie avec des espèces d'oiseaux que nous aimons spontanément soit pour la beauté de leur plumage, soit pour l'excellence de leur chant, soit pour leur compagnonnage agréable, soit pour les qualités humaines qu'ils évoquent ou symbolisent: simplicité, innocence, gaieté, liberté, légèreté, élan, envol spirituel. Autant d'oiseaux pour lesquels nous éprouvons spontanément de l'affection, que nous nous plaisons à observer, à entendre, à nourrir et que nous tentons d'approcher et d'apprivoiser.

Le récit du Sermon aux oiseaux nous présente au contraire des volatiles peu sympathiques, à qui nous parlons volontiers le langage du fusil, que nous voulons éloigner de nos demeures, et que nous pourchassons sans répit. Le récit nous parle d'une multitude de corvidés (corbeaux, corneilles, freux, ramiers, pies, couchas, grolles, selon les variantes des manuscrits). Tous oiseaux de mauvais renoms que personne ne veut voir dans sa cour ou près de son potager. Tous oiseaux qui croassent, criaillent et vous impatientent de leur cri rauque. Tous oiseaux qui se sentent détestés et poursuivis, et qui se sauvent à la moindre alerte.

En Europe le symbolisme du corbeau ainsi que des autres corvidés est presque exclusivement négatif. À cause de sa couleur noire et 
de son aspect nocturne, le corbeau apparaît comme une figure de mauvais augure, lié à la crainte du malheur. Dans l'imagination populaire, il évoque l'image de l'homme avide, rapace, sans scrupule; c'est un messager de mort ${ }^{7}$. C'est vers ces oiseaux de malheur que court le Poverello, émerveillé de ce qu'ils ne s'enfuient pas comme ils font d'habitude. C'est à eux qu'il adresse son premier sermon aux oiseaux. Ce détail n'est pas sans intérêt, comme nous le verrons plus loin.

Le récit rappelle les principaux thèmes qui parcourent les histoires d'animaux rencontrées dans les légendes franciscaines. D’abord il évoque la raison profonde qui motive l'attitude de François envers les oiseaux et les animaux: "son amour était si débordant qu'il témoignait même aux créatures inférieures et privées de raison une grande affection et une grande douceur ». Si cet amour s'exprime ici en douceur et affection, elle prend aussi ailleurs le visage de la pitié, de la compassion, du souci, de l'admiration, du respect, de la nonviolence, de la fraternité.

Cet amour admiratif pour les animaux comme pour les créatures en général s’inscrit dans la nature même des choses, que François trouve et belles et bonnes et utiles. Plus profondément il s'inscrit dans une association symbolique. Moralement, les bêtes peuvent servir de modèles de vie qui doivent inspirer les humains : il les aime pour leur valeur d'édification. Spirituellement, elles peuvent apparaître comme des figures symboliques ou des signes du mystère du Christ: François est plein de douceur envers les agneaux parce qu'ils représentent l'Agneau de Dieu; son amour devient compassion prévenante envers le ver de terre qui lui rappelle le Crucifié qui n'est plus un homme mais un ver. Plus profondément au niveau religieux,

7. J. Chevalier et A. Gheerbrant affirment cependant que traditionnellement en Orient et même en Occident, "ce sont les vertus positives du corbeau sur lesquelles se construit son symbolisme» (Dictionnaire des symboles, Paris, Laffont, 1982, p. 285). 
son amour des bêtes et des choses inanimées jaillit d'un regard encore plus pénétrant: les bêtes et les choses sont des créatures de Dieu. À l'instar de la Bible, François s'intéresse moins à la nature des choses, moins à ce que les êtres sont en eux-mêmes, qu'à leur origine en Dieu. Il considère la réalité non comme nature, mais comme créature. En tant que créé, le monde est entièrement dépendant de Dieu qui, comme créateur, y a laissé ses traces: vestigia Dei. Dans le liber naturae, François voit de magnifiques reflets du Dieu très Saint qui habite son cœur. Chaque créature, bête ou objet inanimé, proclament la gloire, la beauté et l'amour de Dieu. François n'est pas rivé aux choses; son amour des créatures ne s'arrête pas à elles dans un attachement sensuel. Loin de nier leur réalité et leur beauté intrinsèques, il s'en sert d'abord comme tremplin vers Dieu. C'est vraiment son Dieu qu'il aime, admire et glorifie en elles. Son Cantique $d u$ Soleil en est la meilleure illustration. Il aura fallu que son cœur ait été purifié par l'ascèse et l'épreuve pour pouvoir chanter dans un détachement inconditionnel ce chant mystique.

Dans son sermon aux oiseaux, François qualifie ses auditeurs de «créatures de Dieu ». Il les voit dans leur origine; il les voit en Dieu, jaillissant perpétuellement de son sein. Ils font partie de l'immense famille de Dieu, comme d'ailleurs les humains et l'ensemble de l'univers cosmique. S'il n'y a qu'une seule famille, tous les êtres ont entre eux des liens fraternels. Aussi François appelle-t-il les oiseaux: "mes frères les oiseaux». Le beau titre de frère et de sœur sera accolé à toutes les bêtes, à toutes les réalités créées, et principalement aux autres humains. La fraternité est le lien qui relie les êtres et les maintient dans l'unité et l'harmonie. En dernière analyse, François aime les bêtes d'un amour érotique qui s'épanouit en dilection mystique. François aime les animaux et les fleurs pour leur bonté et leur beauté propres, mais il les aime au nom de son amour pour le Christ dont ils sont le symbole et au nom du Créateur dont ils sont des épiphanies glorieuses. Sa motivation n'est ni romantique, ni esthétique, ni écologiste, elle est purement mystique. 
C'est un frère aimant, frère universel et planétaire, qui «plante ses compagnons sur la route et court vers les oiseaux». Que dis-je, un frère? Plus qu'un frère, un homme de Paix. Celano affirme que François «adresse aux oiseaux son salut habituel». Quel est ce salut? «Que le Seigneur vous donne la paix!»Celano déclare ailleurs que François « ouvrait chacun de ses sermons par un souhait de paix avant de transmettre à l'assistance la Parole de Dieu. Cette paix il la souhaitait toujours et avec conviction aux hommes et aux femmes, à tous ceux qu'il rencontrait ou croisait sur sa route » (Vita prima, 23). C'est ce qu'il fait avec les oiseaux. François vient vers eux non comme un chasseur, un maître, un exploiteur, un ornithologue, un amant de la nature, mais comme un homme de paix, tellement pacifié intérieurement qu'il peut faire communier les autres, même les oiseaux et tous les animaux, à sa paix qui n'est autre que celle du Seigneur.

François a fait la paix en lui; il a unifié son âme divisée, il a harmonisé les poussées antagonistes de son être, il a accédé au royaume de la paix à la suite de mille luttes farouches où la chair, c'est-à-dire tout ce qui s'érige contre le soi authentique et contre Dieu, a été vaincue dans ses revendications orgueilleuses et ses prétentions égoïstes. Les peurs inavouées, les forces négatives, les instincts obscurs, les pulsions animales, en un mot, toutes ses bêtes ont été reconnues dans la vérité, regardées avec lucidité, et domestiquées; la paix s'est installée en lui, mais le combat durera jusqu'à la fin.

Les rapports de François avec les animaux, c'est d'abord l'histoire de sa relation avec ses propres bêtes. Ces bêtes qu'on refoule par peur, par civilité ou par conformité à l'ordre social et moral, sont enchaînées au fond de la cage obscure de l'inconscient. Ces bêtes n'ont en soi rien de mauvais; mais parce qu'elles ne sont pas reconnues pour ce qu'elles sont et qu'elles sont tenues en captivité, elles produisent souvent en nous des effets pervers. François n'a pas été apeuré à la pensée du «bestial » en lui. Il ne s'est pas détourné de ses bêtes, ne les a pas niées, ne s'est pas leurré sur leur pouvoir de séduction et de division. Il les a affrontées bien en face; faisant 
confiance en la bonté des bêtes en lui, il les a remises en liberté en les domestiquant, les apprivoisant et en les attelant au chariot de son esprit. Il a muselé et mis frère âne, c'est-à-dire son corps, à son service; il a même pacifié le loup. Qui est ce loup qui terrorise les habitants de Gubbio? Peut-être une bête féroce; partout on crie au loup. Peut-être le podestat de la ville qui écrasait et exploitait les habitants? Peut-être les hérétiques, les Sarrasins ou les ennemis des frères? Peut-être les brigands des grands chemins ou les seigneurs qui volaient et dépouillaient les gens de leurs biens? Peut-être même Frère Élie, Bonaventure, le Pape Jean XXII, qui ont muselé sinon écrasé le mouvement des spirituels ? Toutes les interprétations ont été tentées de cette légende du loup de Gubbio qu'on ne retrouve que dans les Fioretti.

Une chose est sûre, c'est que le loup en François a été apprivoisé; il est devenu lui-même Frère loup. François a fait la paix avec luimême. Entré dans la shalom de Dieu, il peut rayonner la paix et la répandre partout autour de lui. François n'est ni un pacifiste, ni un activiste de la non-violence, ni un médiateur, mais un pacifique, c'est-à-dire un homme qui suinte la paix par tous les pores de son être.

Dans le récit du Sermon aux oiseaux, François quitte ses compagnons et marche vers les oiseaux en homme de Paix. Son contact transforme les désagréables et méprisés corbeaux en oiseaux dociles, attentifs, aimables, joueurs. C'est que François lui-même a domestiqué les corbeaux qui étaient en lui, qui le tenaient éloigné des gens méprisés, symbolisés par les lépreux en particulier dont il avait si grande peur et de qui il se tenait à distance. Ayant domestiqué ses forces de répulsion qui croassaient en lui, il est devenu oiseau luimême: rossignol qui chante, alouette à capuchon, faucon qui veille à minuit. Libéré des attaches terrestres et de la pesanteur charnelle et goûtant à la légèreté de l'âme, François a pris son envol vers les hauteurs célestes. S'il est vrai que l'oiseau est le symbole de l'envol de l'âme immortelle et du vol extatique du mystique, on peut dire que François s'est transformé en oiseau de feu aux ailes de seraphim qui enflamme et soulève nos cœurs balourds, en oiseau du paradis 
qui murmure et chante à nos oreilles terrestres la divine cantate, et en oiseau de l'Ombrie qui, de jour et de nuit, se fait l'infatigable porte-voix de tous les oiseaux en qui et par qui la terre chante sa mélodie à la louange du créateur.

\section{5}

C'est donc un homme de Paix, un homme totalement pacifié et pacifiant qui s'approche des corvidés pour leur prêcher la bonne nouvelle. Si François est entré dans la shalom promise, c'est qu'il a retrouvé son innocence originelle. Cela nous conduit au troisième thème qui parcourt toutes les histoires d'animaux rencontrées dans les biographies franciscaines: c'est le thème de l'innocence primitive, du retour au paradis. Selon notre récit, ce n'est ni par charisme naturel ou talent particulier, ni au contraire par attitude enfantine ou par puérilité des «simples d'esprit», que François parle aux animaux, mais "c'est parce qu'il avait la grâce de la simplicité ». La communion de François avec les animaux est le fruit d'une grâce; la simplicité dont parle Celano est le dépassement de toutes les divisions internes et externes et l'accès à l'unité profonde de l'être, au centre ultime de soi où se fait l'expérience de l'union cosmique. Sur l'arrière-fond des mythes d'origine, il s'agit de la reconquête du paradis perdu, de l'entrée en possession de l'état primordial, du retour à l'harmonie originelle. Et sur l'arrière-fond des mythes de la fin, il s'agit de l'accomplissement eschatologique de la paix cosmique et universelle anticipée dans l'existence spatio-temporelle d'un homme.

François a recouvré la liberté totale: «il va et vient parmi les oiseaux», dit le récit. L'expression «aller et venir» signifie être en liberté. Il va et vient parmi les oiseaux comme Adam au paradis; il les frôle de sa tunique, touche leurs têtes et leurs corps. C'est la fin du règne de la peur; les oiseaux «ne s'enfuient pas comme ils font d'habitude». La sécurité engendre la joie qui est l'état de grâce découlant de la paix retrouvée. Finies la tristesse et la peine! Place à la joie! «Délirant de joie », François va et vient parmi les corvidés sécurisés et convertis qui «expriment à leur façon leur «joie admi- 
rable en allongeant le cou, en déployant leurs ailes, en ouvrant le bec ». Victoire sur la fermeture, la crispation et le repli sur soi. Voici que des êtres recroquevillés et apeurés s'épanouissent et s'ouvrent à la vie totale, grâce à la parole de François.

Le récit de la corneille apprivoisée qui partage la vie des frères, mange avec eux et visite les frères malades, et qui à la mort de François tombe elle-même malade et meurt de tristesse, est une belle illustration de la transformation opérée chez une corneille "détestable » grâce à l'odeur de François. Dans toutes les rencontres de François avec les oiseaux et les autres animaux, le salut se produit: ou bien les animaux sont délivrés de la méchanceté humaine, ou bien ils échappent à la mort, ou bien ils retrouvent la santé, la sécurité et la liberté, ou encore ils éprouvent de la joie de vivre en paix en compagnie des humains.

Autre thème important: celui de l'obéissance des bêtes. Obéissance au commandement de François et obéissance à la parole de Dieu. Dans la plupart des récits, François - tel Adam au paradis - commande aux animaux et ceux-ci lui obéissent. Il ordonne aux fourmis de quitter le vieux chêne, au faucon de le réveiller pour la prière, à la tarentule de ne pas nuire aux frères, à l'araignée de défaire sa toile, à l'âne de se tenir tranquille, au daim d'arrêter de courir, au renard de ne plus toucher aux poules, au loup de faire la paix, à la louve de se départir de sa cruauté. Aux oiseaux, il commande de se taire ou de chanter, de venir ou de s'envoler. Il est à noter que dans les récits son ordre est toujours lié soit à la prédication, soit à la prière communautaire, soit à la contemplation. "Frères oiseaux, commande François, cessez donc de chanter pendant que nous louons le Seigneur. » Dans un autre récit, il ordonne aux hirondelles : «Mes sœurs les hirondelles, vous avez suffisamment parlé; maintenant c'est à mon tour. Écoutez la Parole de Dieu; gardez le silence jusqu'à la fin de mon sermon. Et les petits oiseaux se taisent et ne bougèrent pas de là. » Les oiseaux doivent se taire pour favoriser la prière et la méditation de François et des frères. Devant l'exigence de 
Dieu et de sa parole (prière, oraison, prédication), le rythme naturel doit être suspendu; l'ordre naturel doit être assujetti à l'ordre de la grâce. L'oiseau doit suspendre son activité la plus naturelle non seulement pour rendre possible la prédication et la prière, mais encore pour se mettre lui-même à l'écoute de la Parole de Dieu. Dans le récit du Sermon aux oiseaux, par exception, François n'a pas besoin de faire taire les corvidés, il leur déclare cependant «qu'ils doivent écouter la Parole de Dieu et il les prie humblement d'être attentifs ».

Il est curieux de constater que François se comporte avec les animaux en homme qui commande, alors que dans ses propres écrits, il enjoint à ses frères d'être soumis "à n'importe quel homme de ce monde, et non seulement aux hommes, mais aux bêtes et aux fauves même, les laissant disposer d'eux comme ils veulent autant que d'en-haut leur permet le Seigneur» (Salutation des vertus, 1618). Cette soumission est l'exigence même de la véritable obéissance qui «confond toute volonté propre et tient le corps mortifié pour qu'il obéisse à l'esprit » (14-15). Aussi longtemps que l'homme n'est pas encore entré dans l'obéissance, il doit rester soumis à toute créature; ce n'est qu'une fois devenu obéissant qu'il devient maître du monde et qu'il peut commander aux créatures tout en leur restant soumis. L'obéissance radicale de François prend la forme d'une royale liberté; elle en fait le roi des créatures comme Adam au paradis; il leur commande et elles obéissent.

Les oiseaux obéissent à l'ordre de se taire, d'être attentifs et d'écouter la parole de Dieu. François leur parle et tous les oiseaux "écoutent avec grand respect la parole de Dieu "; et les Fioretti de préciser que les oiseaux «restèrent immobiles jusqu'à ce que Saint François eût fini de prêcher». Notre récit fait l'éloge de la grande «docilité » des oiseaux.

Cette obéissance des animaux et leur écoute de la parole de Dieu sont mises en contraste avec l'incrédulité des êtres humains, sarrasins ou autres, qui, eux, restent sourds et indifférents à la parole de Dieu. Plusieurs histoires d'animaux glorifient cette qualité d'écoute des bêtes dans une visée moralisatrice: dénoncer l'irrespect des êtres doués de raison. Le récit de la procession nocturne des loups et des 
ours à l'Alverne affirme que «le Seigneur s'était servi des animaux pour dénoncer la négligence et la paresse des frères ». La conversion des loups de Greccio et du loup de Gubbio est un reproche vivant à ceux qui refusent de se convertir. Les oiseaux qui ne sèment ni ne moissonnent sont des témoignages éloquents pour ceux qui accumulent pour l'avenir et manquent de confiance en la providence. La légende du sermon aux poissons raconte que François invita les poissons à venir écouter le sermon que les gens d'Agripoli avaient refusé d'entendre. Dans un autre récit, François «se tournant vers la foule de Rome, reproche à ces êtres intelligents et raisonnables (les habitants de Rome) de mépriser la parole de Dieu, alors que des oiseaux privés de raison l'avaient reçue avec grande joie». Et que dire du récit de l'agnelet qui «durant la célébration des Saints Mystères, au moment de l'élévation, ployait les genoux et inclinait la tête en signe de révérence ", sinon qu'il est raconté "pour donner une leçon aux distraits ou indifférents ", et dans un autre cas, pour « inciter les gens à entourer la religion du plus grand respect».

Tous ces textes hagiographiques sont un lointain écho de la cinquième admonition de François où il est écrit: "Considère, homme, dans quelle excellence t'a placé le Seigneur Dieu : il t'a créé et formé à l'image de son Fils bien-aimé quant au corps, et à sa ressemblance quant à son esprit. Et toutes les créatures qui sont sous le ciel, chacune à sa façon, servent leur créateur, le connaissent et lui obéissent mieux que toi ». François est éploré à la pensée que des êtres créés à l'image et ressemblance du Fils soient incapables de reconnaître le Père créateur ou, le connaissant, refusent de lui obéir. Contradiction tragique entre, d'une part, l'excellence humaine et la ressemblance divine et, d'autre part, le refus de servir Dieu. Les êtres humains doivent rougir à la vue des animaux qui, quoique privés de raison, reconnaissent le créateur. Dans son désir aussi douloureux qu'incompressible, François invite, supplie, exhorte, incite, implore, conjure et menace de malédiction éternelle tous ceux qui sont récalcitrants à se tourner vers le Seigneur. Le chapitre 22 de la Première Règle et ses deux Lettres aux fidèles sont les plus émouvants témoins de cet immense et brûlant désir de voir tous les êtres humains honorer, 
servir et aimer le Très Haut et Souverain Seigneur, à l'instar des animaux et autres créatures privées de raison, qui, chacun à sa façon, servent leur créateur et lui obéissent mieux qu'eux tous. L'exemplarité est ici inversée: c'est l'animal qui devient le modèle religieux de l'être humain dont l'excellence est pourtant insurclassable. François semble percevoir l'animal dans son état d'innocence et de non-culpabilité. Ce qui ne l'empêche pas de maudire exceptionnellement les animaux qui adoptent les comportements cruels et déviants : la truie méchante qui dévore le petit frère agneau, créature innocente, qui rappelle le Christ aux hommes; et le rouge-gorge gourmand et repu qui empêche ses petits de se nourrir. Ce qui montre que les animaux ont eux aussi besoin de rédemption.

La relation de François aux animaux découle en tout premier lieu de sa mystique; elle s'enracine dans sa spiritualité. Paul Lachance a montré que, d'après les écrits de François, notamment le chapitre 23 de la Première Règle et les deux Lettres aux fidèles, la spiritualité du mystique d'Assise est trinitaire, fondée sur la divine inhabitation à laquelle tout doit être subordonné, alors que les légendes présentent une spiritualité christologique, centrée sur la vie et la passion du Seigneur ${ }^{8}$. La pauvreté interne et externe que François choisit à la suite de Jésus, est conçue comme un processus de libération des désirs et comme une forme de rejet de tout pouvoir, de toute volonté de puissance et de tout esprit de possession; somme toute, comme un moyen d'émancipation de tout ce qui empêche et détruit la communion avec Dieu et avec les autres. Le chemin de pauvreté conduit à la non-violence, à la paix, à la fraternité universelle et à la communion avec toutes les créatures. Cette complète harmonie cosmique n'est pas le produit d'un sentiment naturel, mais le fruit d'un processus mystique. L'accès à l'harmonie n'est pas d'abord le fruit d'un

8. P. Lachance, "Mysticism and Social Transformation, According to the Franciscan Way ", dans J.K. Ruffing, dir., Mysticism and Social Transformation, Syracruse, Syracruse University Press, 2001, p. 55-75. 
amour spontané pour les créatures, mais le produit d'un processus de purification, d'ascèse, de renoncement extrême. Il faut comprendre les récits légendaires d'animaux dans cette perspective, comme liés à cette mystique ascétique et au style de vie érémitique qui obligeait François, le citadin, à s'adapter à un environnement terrifiant, hostile et hanté, et à la compagnie forcée des animaux sauvages. François, l'anachorète, a dû élargir jusqu'aux bêtes féroces son approche fraternelle.

La vie dans les retiros a forcé l'ermite d'Assise à la convivialité avec les bêtes. François s'est vu contraint de maîtriser ses craintes, d'apprivoiser les bruits de la forêt et de se familiariser avec les mœurs des animaux; de leur côté, les bêtes des alentours ont dû apprendre à surmonter la peur des humains. À n'en pas douter, les premières expériences érémitiques de François ont été le stimulus originel dans l'éclosion de son amour et de sa sympathie pour les animaux et dans l'élaboration d'une vision positive des créatures, en contraste avec la suspicion pathologique largement répandue envers le monde sensible, vu comme source de tentation diabolique et de perversion morale. Frères les animaux prenaient graduellement place dans son esprit à côté de Frère soleil, de Frère feu, de Sœur eau et de Mère terre. L'effort d'adaptation à ce monde sauvage et hostile devenait partie prenante de son combat spirituel. La convivialité de François envers les animaux est donc inséparable de son expérience et de son idéal de vie érémitique. Dans sa grotte, François refait l'expérience de Jésus qui, au désert, «vivait avec les bêtes sauvages » (Mc 1,13).

François, l'ermite. C'est un mystique cosmique qui descend des hauteurs de grottes solitaires pour entreprendre une campagne d'évangélisation. Que s'est-il passé dans sa vie qui ait pu opérer pareil changement? François a été longtemps hanté par la pensée et le désir de se retirer entièrement du monde pour mener une vie d'anachorète; et il en avait parlé souvent à ses compagnons. En vérité, une seule chose l'empêchait d'opter résolument pour la solitude : l'exemple du Christ qui, ayant quitté la quiétude du sein du Père, avait passé sa vie sur les routes de Palestine à annoncer le Royaume. 
Pour trancher doutes et tergiversations, François avait l'habitude d'ouvrir l'Évangile au hasard; il obéissait aveuglément au passage qui tombait sous ses yeux. Cette fois-ci, c'était en 1212, il s'en remit au bon jugement de deux personnes dont il estimait la qualité du discernement: frère Sylvestre et soeur Claire. C'est d'après leurs avis qu'il marcherait, ainsi en avait-il décidé. Ils lui répondirent: « NotreSeigneur veut que tu continues de prêcher, car Dieu ne t'a pas appelé seulement pour ton propre salut, mais aussi pour le salut des autres ». François obtempéra aussitôt et se mit en route vers Bevagna pour entreprendre une campagne d'évangélisation. Mû par son immense désir de partager son expérience de Dieu, de susciter la fraternité cosmique et de faire communier les bêtes à son grand mouvement d'adoration, il décide de ne plus prêcher seulement aux hommes mais aussi aux animaux. C'est alors qu'il plante là ses compagnons et court vers les oiseaux pour leur adresser la parole. Dans son intuition originale, marquée au coin d'un certain littéralisme, il découvre le sens cosmique du commandement du Seigneur: «Allez enseigner toutes les créatures.» (Mc 16,15) Non seulement les êtres humains mais toutes les créatures sous le soleil. C'est ainsi que, pour la première fois, il s'adresse aux oiseaux. Le récit du Sermon aux oiseaux précise que François «s'accuse de négligence pour n'avoir pas encore prêché aux oiseaux et qu'à partir de ce jour, il ne manquait pas d'exhorter tous les oiseaux, tous les animaux, les reptiles et même les créatures insensibles à louer et aimer le Créateur ».

Nous assistons ici à un changement de paradigme dans la tradition spirituelle relative aux rapports des saints avec les animaux. Jusque-là, le rapport de François était lié à son expérience mystique de type érémitique qui en était pour ainsi dire le Sitz im Leben. Dorénavant s'ajoute un nouveau contexte, celui de la vie apostolique. Aussi de très nombreux récits d'animaux prennent-ils place au cœur des tournées d'évangélisation. La convivialité de l'ermite avec les bêtes s'épanouit en source d'évangélisation des animaux. Eux aussi font partie de la création qui «attend avec impatience d'être libérée de l'esclavage et de la corruption pour avoir part à la liberté et à la gloire des enfants de Dieu» (Rm 8,20-21). Eux aussi font 
partie du mystérieux dessein de Dieu sur le monde. Au début, les animaux étaient près d'Adam dans l'Éden, à la fin ils seront de quelque manière près de François dans le Règne. Les animaux ont à être évangélisés: ce ne sont pas de viles machines vivantes, mais des créatures de Dieu appelées à partager une destinée sublime.

Évangéliser les animaux, qu'est-ce à dire ? S'agit-il de les domestiquer, de les dompter, de les dresser, d'en faire des bêtes savantes, de les extraire du règne animal pour les exhausser au plan humain? S'agit-il d'ouvrir des S.P.C.A. ${ }^{9}$ spirituels où les animaux seraient entraînés à ployer les genoux devant le Saint-Sacrement comme dans la légende franciscaine, et les oiseaux à chanter des hymnes à la louange de Dieu? S'agit-il de parler aux animaux comme on parle à son toutou et d'apprendre quelques mots religieux à son perroquet?

Peut-être trouverons-nous la réponse dans le Sermon aux oiseaux? Quelle parole évangélisatrice François adresse-t-il aux corvidés? «Mes frères, les oiseaux, vous avez bien sujet de louer votre créateur, car il vous a donné des plumes pour vous vêtir, des ailes pour voler et ce dont vous avez besoin pour vivre». Cette invitation à la louange s'inscrit dans la profondeur de la spiritualité de frère François et de son élan mystique. François d'Assise, l'inconditionnel de la louange. Il n'y a pas de situation aussi négative qu'elle puisse être, comme la mort, la souffrance et le rejet, qui ne soit occasion de chanter la gloire du créateur. François est parvenu à une telle dépossession de soi, à une telle pauvreté purificatrice qu'il a une conscience vive de se recevoir totalement de Dieu en toute situation. Aussi la seule attitude conséquente qui s'impose, c'est la reconnaissance qui se fait action de grâce et louange.

Pour bien comprendre l'invitation à la louange adressée aux oiseaux, il faut la situer sur l'arrière-fond du Cantique des créatures où elle appartient naturellement. C'est un François aveugle, au corps émacié, déchiré par la souffrance et sentant venir la mort qui ex-

9. Société protectrice canadienne des animaux. 
prime le tréfonds de son âme dans ce Cantique, qui commence par une invitation universelle à l'inaccessible louange:

Très-Haut, Tout-Puissant, bon Seigneur,

À Toi sont la louange, la gloire et l'honneur et toute bénédiction.

À Toi seul ils conviennent, et nul homme n'est digne de te nommer.

Cette phrase introductrice reprend le thème du chapitre 23 de la Première Règle et de l'Exhortation à la louange de Dieu composée par François lui-même. Dieu seul est digne de louange parce qu'il n'y a aucun bien en-dehors de lui: «lui seul est le bien plénier, le bien universel, le bien total, le vrai et souverain bien»(1 Reg 23,9), qui «ne nous a fait et ne nous fait que du bien» (1 Reg 23,8). Aussi l'âme de François, libérée de toute attache aux vanités et aux illusions est-elle toute tendue vers le seul Bien. La louange n'est pas seulement un chant de sa bouche, mais la respiration de tout son être.

Pourtant il sait que sa louange ne peut atteindre le divin Bien. Lui, François, se sent si petit, si misérable, si pécheur et Dieu, lui, est si haut. "Indigents et pécheurs que nous sommes tous. Nous ne sommes pas dignes de te nommer" (Première Règle 23,5). Le Cantique reprend le thème: "nul homme n'est digne de te nommer ". C'est l'expérience de l'inaccessible louange. Pour louer, il faut d'abord nommer. Malgré son indignité et son incapacité, François n'a de cesse et maintient son élan vers l'impossible saisie, l'inaccessible louange.

Seul, il se sent impuissant, mais peut-être qu'en se joignant à toute l'humanité, sa louange touchera-t-elle le port inabordable. C'est ainsi qu'au chapitre 23 de la Première Règle, il exhorte "tous les peuples, les races, les tribus et les langues, toutes les nations et tous les hommes, partout sur la terre qui sont et seront » à se joindre à sa louange. Dans le Cantique des créatures, il élargit son invitation à toutes les créatures: "Loué sois-tu, mon Seigneur, avec toutes tes créatures. » Cette fois, François introduit toutes les créatures dans son mouvement ascensionnel vers le Très-Haut. Le «avec toutes les créatures" signifie que non seulement François se met du côté des créatures, mais qu'il les entraîne dans son envol de louange. Il a 
découvert son lien cosmique. Le monde n'est plus objectivement en face de lui; il est subjectivement en lui. Il a noué un lien de communion si fort avec toute la création qu'il peut appeler toutes choses, frères et sœurs. Il ne peut entrer dans l'inaccessible louange qu'en portant le monde dans son élan. La communion à la totalité se fait au-dedans: dans son effort, la "sève du monde " (Teilhard de Chardin) s'épanouit en louanges au Créateur.

Des créatures, François attend qu'elles l'aident à louer le TrèsHaut. Il leur demande « de lui révéler quelque chose du Créateur, de sa puissance, de sa bonté, de sa beauté: il leur demande de lui souffler la louange inaccessible ${ }^{10} »$. De la communion (avec) on passe maintenant à la médiation (pour) : "Loué sois-tu mon Seigneur, pour la lune, les étoiles, le vent, l'eau, la pluie, la terre parce que de Toi Très-Haut, il porte le signe ». Cette médiation de révélation cosmique aide François à nommer le Très-Haut et à le louer.

Dans le Sermon aux oiseaux, François nomme ce que le Cantique omet de mentionner. Dans ce sermon comme d'ailleurs dans son Exhortation à la louange de Dieu, il invite les oiseaux et à travers eux, tous les animaux à entrer dans sa louange cosmique. François a besoin que sa louange soit portée par le chant de oiseaux. Il entraîne tous les oiseaux et animaux avec lui dans son mouvement vers le Très-Haut. Ainsi le récit du Sermon aux oiseaux et les histoires d'animaux que raconte la légende franciscaine, évoquent une dimension essentielle de l'âme de François et un trait spécifique de sa spiritualité.

10. LeClerc, Le Cantique des créatures, p. 60. 


\section{RÉSUMÉ}

Cet article propose une série d'évocations sur le rapport de François d'Assise avec les animaux, rapport qui est abordé ici à partir du récit du Sermon aux oiseaux qui s'impose par son ancienneté, son autorité et sa popularité. Le récit contient les principaux thèmes évoqués dans la cinquantaine d'histoires d'animaux rencontrés dans le Légende franciscaine: innocence originelle, harmonie, soumission des animaux, maîtrise des bêtes en soi-même, valeurs symboliques. Le rapport de François aux animaux n'est ni romantique, ni écologique mais mystique. Ce n'est qu'après avoir passé à travers les exigences d'une rude ascèse et d'une dépossession radicale qu'il redécouvre la portée épiphanique de la nature et le lien fraternel qui l'unit aux choses et aux bêtes. L'expérience érémitique a été le premier déclenchement d'un tel sentiment de fraternité avec les bêtes qui a trouvé dans le vita apostolica un second lien d'inscription. Le rapport de François aux animaux doit être compris sur l'horizon de son fameux Cantique des créatures qui est l'expression la plus puissante de sa vision mystique de l'interconnexion de toutes réalités créées.

\section{ABSTRACT}

This article proposes a series of accounts describing the relationship between Francis of Assisi and animals, a relationship which is presented in a recognized and ancient text called the Sermon aux oiseaux (Sermon to the Birds). This depiction is filled with the major themes contained in the animal stories of the Légende franciscaine (Franciscan Legend): original innocence, harmony, compliance of animals, control over one's inner beasts, symbolic standards. The relationship of Francis of Assisi with animals is not romantic, nor ecological, but it is primarily a mystical affinity. Only after had he trod the path of asceticism and of radical poverty, will he rediscover the radiance of nature and its fraternal link with animals and with all things. This eremitical experience has been the first in its kind. It has served to awaken a sense relationship between man and animals and was therefore written in the vita apostolica. The relationship between Francis of Assisi and animals, must be understood in the context of one of his important works, the Cantique des créatures (Song to the Creatures), which is the most wonderful expression of the mystical vision he had concerning the interconnectedness of all created things.

(C) Revue Théologiques 2002. Tout droit réservé. 